\title{
Relation of latency of galvanic skin reflex to frequency of the electroencephalogram
}

WALTER W. SURWILLO

UNIVERSITY OF LOUISVILLE SCHOOL OF MEDICINE

Galvanic skin reflexes (GSRs) were evoked in 42 healthy males by short, supra-threshold tones presented at random and without warning while electroencephalograms (EEGs) were recorded. Period (reciprocal of frequency) of EEG, in the interval between presentation of stimulus and initiation of GSR, was correlated with GSR latency. A statistically significant but low positive correlation of 0.26 suggested that, in contrast to results with voluntary responses, latency of GSR is largely independent of cortical (EEG) influences.

Several investigations (Denier van der Gon \& Van Hinte, 1959; Mundy-Castle \& Sugarman, 1960; Surwillo, 1963, 1964a) have examined the relation of frequency of the human electroencephalogram (EEG) to the temporal organization of behavior. These studies reveal that latency of voluntary responses and time taken to perform simple tasks are related to period of alpha rhythm (period equals reciprocal of frequency), when such activity is present in the EEG. In the main, they show that slow-responding persons have waves of longer period (lower frequency) in their EEGs than fast-responding persons. Positive correlations have also been observed between simple reaction time and EEG period in individual Ss (Surwillo, 1963). In five Ss whose EEGs could be synchronized to a highintensity light that was flashing at frequencies in the alpha rhythm band while they were tested on a reaction task, Surwillo (1964b) reported that correlations between reaction time and period of flash were positive.

Although these studies suggest that period of EEG waves is an important factor in the temporal organization of behavior, the findings cited were limited to response systems associated with voluntary activities. Thus, we cannot say whether the same principle applies, for example, to speed of responses in other systems. Our purpose in the present study was to provide some answer to this question by determining whether latency of an autonomic response, the galvanic skin reflex (GSR), is correlated (positively) with period of the EEG.

Method

Ss were 42 healthy males drawn from a larger group of persons whose EEGs were recorded during a preliminary practice session. The $42 \mathrm{Ss}$ included only those whose records showed well-developed alpha rhythm $(25 \mu \mathrm{V}$. peak-to-peak or better), roughly $75 \%$ or more of the time.

EEGs were picked up from four sponge-type surface electrodes placed according to the "10-20" International System (Jasper, 1958). Electrodes over $\mathrm{O}_{1}, \mathrm{O}_{2}, \mathrm{Fp}_{1}$, and $\mathrm{Fp}_{2}$, were held in place by means of a headgear fashioned from strips of lastonet bandage. A ground electrode on the doraal surface of the neck, approximately over the sixth cervical vertebra, was held in place with adhesive tape. Four channels of bipolar EEGs were recorded: $\mathrm{O}_{1}-F p_{1}, \mathrm{O}_{2}-F p_{2}$, $\mathrm{O}_{1}-\mathrm{O}_{2}$, and $F p_{1}-F p_{2}$. The lead placements were like those used earlier by Surwillo (1963, 1964a). They were originally chosen because we were interested primarily in alpha activity; with them, electrodes on each hemisphere were lined up along the voltage gradient of the alpha rhythm (Davis, 1961).

GSRs (endosomatic) were recorded by means of $\mathrm{Ag}-\mathrm{AgCl}$ electrodes, of the type described by Quilter \& Surwillo (1966), attached to the palm (active electrode) and to the ventral surface of the forearm (reference electrode). The areas were selected to avoid constriction of blood flow and, to insure low electrical resistance at the reference electrode, a sponge soaked in $5 \%$ aqueous solution of $\mathrm{NaCl}$ was kept in contact with the skin of the forearm for at least $15 \mathrm{~min}$. before the electrode was attached. Electrodes were connected to a DC amplifier with high-impedance input that was calibrated for a deflection sensitivity of $2 \mathrm{mV} / \mathrm{cm}$. GSRs were traced out on the chart of a polygraph along with the EEGs.

The test procedure was carried out with S lying on a bed, with his eyes closed, in a darkened room. A short (0.3 sec.) 250 cycle tone was presented, from time to time and without warning, over a near-by loudspeaker. $\mathrm{S}$ held a response key in his right hand and was told to press the button as quickly as possible whenever he heard a tone. The 250 cycle tone, which also served as a stimulus for the GSR, was well above threshold, but not so loud as to evoke a startle response. It almost invariably elicited a GSR when it was presented; only very rarely in this study did the GSR become completely habituated and disappear during a trial. Since Davis (1930) reported that latency of the GSR is not a function of stimulus intensity, the level of the tone was not critical in the present experiment.

Stimuli were triggered by the experimenter who watched the EEGs as they were recorded and attempted to trigger stimuli when well-defined waves were recorded, and to avoid presenting them during musclepotential artifacts, eye and eyelid movements, and the like. A separate channel recorded the presentation of the stimuli on the chart along with EEG and GSR tracings. The 250 cycle tone was presented at random a total of 30 times in the course of a $10 \mathrm{~min}$. interval.

In measuring latency of the GSR, the first 10 trials of the test were arbitrarlly discarded. Only negative deflections $\geqq 0.2 \mathrm{mV}$, which were referred to by Forbes (1936) as "a" responses, were considered to be GSRs. Latency was determined by measuring 
the lineal distance between onset of stimulus and a point corresponding to the initial negative deflection of the trace, and converting this value into units of time. A trial was rejected if artifacts such as muscle potentials or an electrocardiogram obscured the GSR tracing.

Period of the EEG was measured according to a set of rules that was developed and reported earlier by Surwillo (1963). Measurements were averages of the period of individual waves recorded in the interval between presentation of tone and initiation of GSR. As in the exrlier investigations, our rationale for observing EEG period in this particular interval was based on the logic thata timing signal must be temporally contiguous with the interval of time that it defines. Attenuation of the EEG due to the stimulus, or "blocking" as it is called, frequently produced such severe reduction in amplitude of the tracing that measurement of EEG period became impossible. In order to cope with this problem systematically, all trials where attenuation produced gaps in measurement of waves for $25 \%$ or more of the time between stimulus and GSR were rejected. This procedure assured that determinations of EEG period were strictly according to rule, and that the values reported for each trial were based on a representative number of waves. On the other hand, this rule was largely responsible for the fact that an average of $63 \%$ of the data collected, or 12.6 out of 20 trials for each S, could not be measured. Results and Discussion

Average values of GSR latency and EEG period were computed for each of the $42 \mathrm{Ss}$; they were based on an average of only 7.4 trials per $S$, mainly because of EEG "blocking" as explained earlier. Mean GSR latency for the group was 1.75 sec., with a range of 1.36-2.29 sec., and mean EEG period was $101.6 \mathrm{msec}$., with a range of 82.9-131.3 msec. Expressed in terms of frequency (cycles per second), the latter values correspond to a mean of $9.84 \mathrm{c} / \mathrm{sec}$., and a range of 7.62-12.06 c/sec. The coefficient of correlation between GSR latency and EEG period was 0.26, which is low but statistically significant at the 0.05 level.

Latency of S's voluntary responses-the reaction time-was also determined and average reaction time was computed for each $S$. Correlation of these values with the means of GSR latency yielded a coefficient of -0.02 , which revealed that reaction time and latency of the GSR were not related.

While our findings show that GSR latency is related to period of the EEG, the slze of the correlation is disappointingly low. The coefficient of determination $\left(r^{2}\right)$ reveals that period of EEG can account for barely $7 \%$ of the total variance in GSR latency. This is in marked contrast to studies of reaction time where correlations in the $0.70^{\prime} \mathrm{s}$ have been reported (Surwillo, $1963,1964 a)$. Since the frequency range of 7.62-12.06 c/sec. reported in this study covers the major portion of the alpha-rhythm band of 8-13 c/sec., it seems unlikely that our low correlation could be the result of restriction in range of this measure.
Because peripheral speed of conduction of the GSR is slow, with a rate in the neighborhood of only $1.0 \mathrm{~m} / \mathrm{sec}$. (Broughton et al, 1965; Wang, 1957), conduction time could account for a substantial percentrge of GSR latency. Differences in stature and the accompanying differences in length of conduction pathway could, therefore, account for a portion of the variance in latency of GSR which, in turn, could result in a lower correlation with EEG period. Unless length of pathway accounted for a very large portion of the total variance, however, its effect in the present investigation would be almost negligible. Thus, for example, if $50 \%$ of total variance were accounted for by variance in length of conduction pathway, the correlation of 0.26 reported in the present investigation would increase to only 0.37 by holding pathway length constant.

Although our data suggest that GSR latency is largely independent of cortical (EEG) influences, a note of caution should be sounded concerning interpretations of the present findings. Electrode placements used in this experiment were chosen because they had been employed in earlier studies relating EEG period and latency of voluntary response. Although the rationale for recording from the same areas in the present study was based on the assumption of a single timing mechanism controlling all behavior, it is possible that timing of GSR is mediated by a separate mechanism. To settle this question, other cortical areas will need to be explored.

\section{References}

Broughton, R. J., Poire, R., \& Tassinari, C. A. The electrodermogram (Tarchanoff effect) during sleep. EEG clin. Neurophysiol., $1965,18,691-708$.

Davis, J. F. Monopolar methods in electrophysiology. In L. E.. Slater (Ed.), Proceedings of the interdisciplinary clinic on the instrumentation requirements for psychophysiological research. New York: Foundation for Instrumentation Education and $R e$ search, 1961. Pp. 91-96.

Davis, R. C. Factors affecting the galvanic reflex. Arch. Psychol., New York. 1930, No. 115.

Denier van der Gon, J. J., \& Van Hinte, N. The relation between the frequency of the alpha-rhythm and the speed of writing. $E E G$ clin. Neurophysiol., 1959, 11, 669-674.

Forbes, T. W. Skin potential and impedance response with recurring shock stimulation. Amer. J. Physiol., 1936, 117, 189-199.

Jasper, H. H. The ten twenty electrode system of the International Federation. EEG clin. Neurophysiol., 1958, 10, 371-375.

Mundy-Castle, A. C., \& Sugarman, L. Factors influencing relations between tapping speed and alpha rhythm. EEG clin. Neurophysiol., 1960, 12, 895-904.

Guilter, R. E., \& Surwillo, W. W. A simple method for preparing silver-silver chloride electrodes for recording of skin potential. Amer. J. Psychol., 1966, 79, 309-313.

Surwillo, $w$. $w$. The relation of simple response time to brain wave frequency and the effects of age. EEG clin. Neurophysiol., $1963,15,105-114$.

Surwillo, $w$. $w$. The relation of decision time to brain wave fre quency and to age. EEG clin. Neurophysiol., 1964a, 16, 510-514.

Surwillo, $w$. $w$. Some observations on the relation of response speed to frequency of photic stimulation under conditions of EEG synchronization. EEG clin. Neurophysiol., 1964b, 17, 194-198.

Wang, G. H. The galvanic skin reflex. A review of old and recent works from a physiologic point of view. Part I. Amer. J. phys. Med., 1957, 36, 295-320. 